\title{
Mechanism of strain relaxation by twisted nanocolumns revealed in AIGaN/GaN heterostructures
}

V. P. Kladko, A. V. Kuchuk, N. V. Safryuk, V. F. Machulin, A. E. Belyaev, H. Hardtdegen, and S. A. Vitusevich

Citation: Appl. Phys. Lett. 95, 031907 (2009);

View online: https://doi.org/10.1063/1.3184569

View Table of Contents: http://aip.scitation.org/toc/apl/95/3

Published by the American Institute of Physics

\section{Articles you may be interested in}

Internal strains and crystal structure of the layers in AIGaN/GaN heterostructures grown on a sapphire substrate

Journal of Applied Physics 105, 063515 (2009); 10.1063/1.3094022

An influence of the local strain on cathodoluminescence of $\mathrm{GaN} / \mathrm{Al}_{\mathrm{x}} \mathrm{Ga}_{1-\mathrm{x}} \mathrm{N}$ nanowire structures Journal of Applied Physics 120, 194304 (2016); 10.1063/1.4968004

Optical and structural study of deformation states in the GaN/AIN superlattices

Journal of Applied Physics 122, 155302 (2017); 10.1063/1.4999175

Effect of well/barrier thickness ratio on strain relaxation in GaN/AIN superlattices grown on GaN/sapphire template

Journal of Vacuum Science \& Technology B, Nanotechnology and Microelectronics: Materials, Processing, Measurement, and Phenomena 35, 062902 (2017); 10.1116/1.4999468

Strain-induced polarization in wurtzite III-nitride semipolar layers

Journal of Applied Physics 100, 023522 (2006); 10.1063/1.2218385

Strain relaxation in short-period polar GaN/AIN superlattices

Journal of Applied Physics 106, 013526 (2009); 10.1063/1.3168431

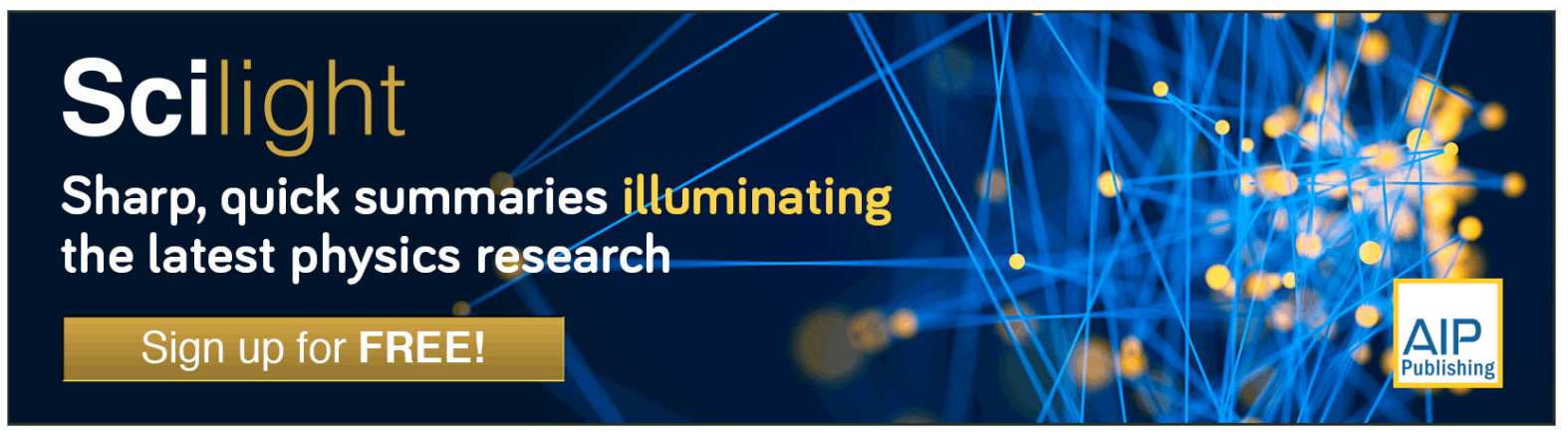




\title{
Mechanism of strain relaxation by twisted nanocolumns revealed in AIGaN/GaN heterostructures
}

\author{
V. P. Kladko, ${ }^{1}$ A. V. Kuchuk, ${ }^{1}$ N. V. Safryuk,${ }^{1}$ V. F. Machulin, ${ }^{1}$ A. E. Belyaev, ${ }^{1}$ \\ H. Hardtdegen, ${ }^{2}$ and S. A. Vitusevich ${ }^{2, a}$ \\ ${ }^{1}$ V. Lashkaryov Institute of Semiconductor Physics, NAS of Ukraine, Kiev 03028, Ukraine \\ ${ }^{2}$ Institut für Bio- und Nanosysteme, Forschungszentrum Jülich, D-52425 Jülich, Germany
}

(Received 6 May 2009; accepted 26 June 2009; published online 20 July 2009)

\begin{abstract}
The structural properties of $\mathrm{AlGaN} / \mathrm{GaN}$ heterostructures grown by metal organic chemical vapor deposition on sapphire substrates with different thicknesses were studied by high-resolution $\mathrm{x}$-ray diffraction. The relation between the deformations and dislocation densities in the layer and substrate was established. The dependence of the system's curvature on the lattice mismatch, caused by different fractions of nanoblock twists with respect to the $c$-plane, was determined. A mechanism of elastic strain relaxation was proposed. () 2009 American Institute of Physics.
\end{abstract}

[DOI: $10.1063 / 1.3184569$ ]

III-nitrides are materials of rapidly increasing importance for the semiconductor industry for a broad range of applications from light-emitting and laser diodes to hightemperature and high-power electronic devices. ${ }^{1}$ In spite of recent achievements, the key limitation is the growth technology, because the large mismatch in lattice parameters between the sapphire substrate and thin film, as well as the difference in their coefficients of thermal expansion, results in a large concentration of structural defects with dislocation densities of the order of $\approx 10^{8} \div 10^{10} \mathrm{~cm}^{-2}$ and in significant residual deformations. ${ }^{2,3}$ The III-nitride layers grown on an $\mathrm{Al}_{2} \mathrm{O}_{3}$ (0001) substrate have predominantly a mosaic structure consisting of $c$-oriented nanocolumns. ${ }^{4}$ However, up to now, the physical mechanism explaining the features in the mosaic structure of III-V alloys has not been established, which has considerably delayed progress in this field of research. In order to solve the problem, the influence of the deformation state on the structural and electrical properties of multilayered nitride heterostructures, on their mosaic structure as well as on defects caused by processes of elastic strain relaxation needs to be studied in detail. X-ray diffraction (XRD) is the most effective method of obtaining structural parameters, the magnitude of elastic strain deformation, and the composition of the nitride structures. ${ }^{5}$ In this letter, we investigate the influence of dislocations (dislocation density, type of dislocations, and the law of their distribution) on the deformation conditions of $\mathrm{Al}_{x} \mathrm{Ga}_{1-x} \mathrm{~N} / \mathrm{GaN}$ heterostructures grown on substrates with different thicknesses. We propose the relaxation mechanism of strain in the structures by twisted nanocolumns and demonstrate that the experimentally obtained results are in good agreement with the proposed model.

The $\mathrm{AlGaN} / \mathrm{GaN}$ heterostructures under investigation were grown by metal organic chemical vapor deposition on (0001) sapphire substrates with thicknesses of 3 and 0.45 $\mathrm{mm}$. After the deposition of a 3- $\mu \mathrm{m}$-thick GaN buffer layer, the $\mathrm{Al}_{0.3} \mathrm{Ga}_{0.7} \mathrm{~N}$ layer with a thickness of $30 \mathrm{~nm}$ was grown, followed by a $4 \mathrm{~nm} \mathrm{GaN}$ cap layer. The misorientation of the substrate surface did not exceed 30 angular minutes. The

\footnotetext{
${ }^{a)}$ Electronic mail: s.vitusevich@fz-juelich.de. On leave from the Institute of Semiconductor Physics, NASU, 03028 Kiev, Ukraine.
}

structural quality of the samples was investigated by highresolution XRD., ${ }^{6,7}$ The experimental setup allows us to obtain two cross sections of the reciprocal lattice nodesorthogonal to the diffraction vector, the $\omega$-scan and parallel to it, the $\omega / 2 \theta$ scan. The measurements were performed on "PANalytical X'Pert PRO MRD" equipment using symmet-

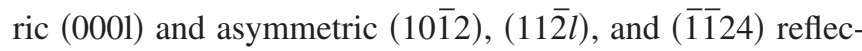
tions. The asymmetric Bragg geometry allows us to combine the properties of symmetric Bragg and Laue diffractions, giving us the opportunity to study the effects of interplane spacing changes and twists of atomic plane separately by an analysis of the intensity distribution in the $q_{x}, q_{y}$ coordinate system, directed along and orthogonal to the $\boldsymbol{H}$ vector, respectively. Azimuthal $\varphi$-scanning for asymmetrical $(11 \overline{2} l)$ reflections was used to measure the misorientation of $\mathrm{GaN}$ blocks with respect to the substrate plane.

The macrodeformations induced by bending the sample are important characteristics of the GaN layer. The curvature radius can be determined by measuring the deviation of the angle of a sapphire reflection at a linear scanning of the sample under a constant $\mathrm{x}$-ray beam. ${ }^{8}$ Since the layer thickness is much smaller than the substrate thickness, the curvature radius of the layer is assumed to be the same as that of the substrate. The influence of the dislocation network on the Bragg diffraction results in the broadening of the diffraction pattern along the direction orthogonal to the diffraction vector $\boldsymbol{H}$. This effect was resolved for all types of investigated structures. However, the dislocation networks do not change the intensity distribution along the diffraction vector.

A Williamson-Hall plot was used to obtain information about microdeformation inside mosaic blocks, the sizes of these blocks (vertical and lateral coherence length), and the tilts and twists of the blocks. ${ }^{9}$ The sizes of the coherence length along the growth direction (height of the blocks) of GaN layers grown on the thick substrate are twice as large as those of the layers grown on the thin substrate. At the same time, the vertical coherence lengths in AlGaN layers are the same for both cases and are equal to their thickness. The deformation parameters and sizes of the coherent length in $\mathrm{GaN}$ layers are correlated with the density of the edge dislocations. The densities of the screw and edge dislocations with Burgers vector close to the orthogonal direction with 
TABLE I. Structural parameters obtained from the XRD data.

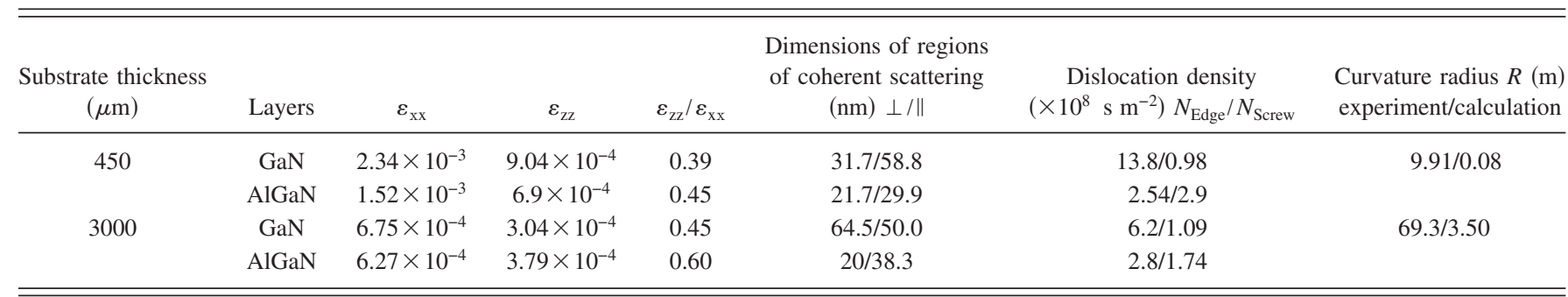

respect to the growth plane are practically the same for GaN layers of the heterostructures. The components of the deformation tensor $\varepsilon_{\mathrm{zz}}$ for $\mathrm{GaN}$ layers grown on the thin substrate are three times as large as those grown on the thick substrate (Table I). The same relation holds for AlGaN layers.

The measured and calculated values of the curvature radius for both structures are different. Such disagreement was the main reason for the detailed study, since it was not possible to explain the difference as usual by the effects of the strain elastic relaxation and the dislocation density. The mismatch of planes in heterostructures is caused by the twist of the corresponding layers with respect to the substrate and each other. The screw component of dislocation with Burgers vector parallel to the interface and edge dislocations with Burgers vectors $\boldsymbol{b}_{\boldsymbol{E}}=1 / 3\langle 1120\rangle$ result in the twisting of the planes. This is a direct verification of the theoretical concept by which the macromismatch of layers with substrate is caused by the dislocation network and deviation of the crystal's surface from the regular crystallographic orientation. ${ }^{10}$

The decreasing difference between $\varepsilon_{\mathrm{xx}}$ and $\varepsilon_{\mathrm{zz}}$ components for the sample grown on the thick substrate suggests that in this case the structure is untypical (the typical structure usually has linear dislocation ensembles with Burgers vectors perpendicular to the interface), and it has more random distribution of dislocations as a function of thickness. At the same time, the local variation in interplane spacing for planes perpendicular to the film plane is significantly larger than that parallel to it.

The mismatch of lattice parameters in the interface plane for $\mathrm{GaN}$ and $\mathrm{Al}_{2} \mathrm{O}_{3}$ is the same $(\Delta a / a)_{\|}=\left(a_{\mathrm{GaN}}\right.$ $\left.-a_{\mathrm{Al}_{2} \mathrm{O}_{3}}\right) / a_{\mathrm{Al}_{2} \mathrm{O}_{3}}=-0.33$, where $a_{\mathrm{GaN}}=0.31876 \mathrm{~nm}$ and $a_{\mathrm{Al}_{2} \mathrm{O}_{3}}=0.4758 \mathrm{~nm}$. ${ }^{11}$ Such mismatch with coherent growth

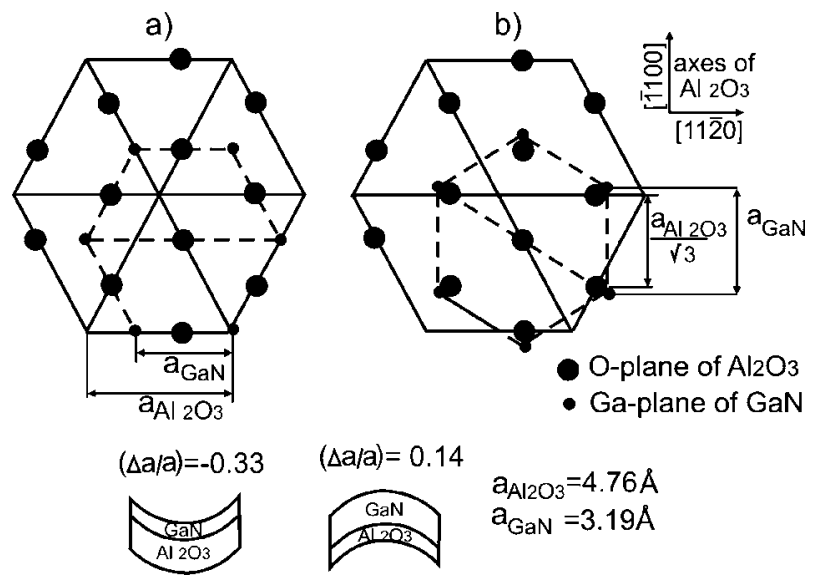

FIG. 1. Schematic presentation of atom arrangements in growth plane for the case of a (0001) GaN layer, grown on sapphire (0001) at mismatch of lattice parameters equal to -0.33 (a) and 0.14 (b). results in macrobending of the substrate-epilayer system (concave from the layer side). However, from experimental results we found that the system is convex. This fact remains to be explained.

We suggest that the abovementioned effect is caused by the arrangement of $\mathrm{GaN}$ blocks on the interface plane at different (opposite sign) lateral mismatch. The value of such mismatch $(\Delta a / a)_{\|}=\left(a_{\mathrm{GaN}}-a_{\mathrm{Al}_{2} \mathrm{O}_{3}}\right) / a_{\mathrm{Al}_{2} \mathrm{O}_{3}}=0.14\left(a_{\mathrm{GaN}}\right.$ $=0.31876 \mathrm{~nm}$ and $\left.a_{\mathrm{Al}_{2} \mathrm{O}_{3}}=0.2749 \mathrm{~nm}\right)$ can be obtained by rotation of the $\mathrm{GaN}$ cell by $30^{\circ}$ around the [0001] axis (Fig. 1) with respect to the sapphire cell. In the experiment, the asymmetrical reflections $(11 \overline{2} l)$ from the GaN layer and substrate are fixed at different azimuthal positions of the sample (Fig. 2). It was established that the lateral mismatch of $\mathrm{GaN}$ and sapphire cells is about $25.8^{\circ}$ on the thin substrate (but it is not $30^{\circ}$ as follows from Fig. 1) and $31^{\circ}$ for the structures grown on a thick substrate. It should be noted that the large dislocation density with the edge and screw segments (Burgers vector is parallel to the interface plane) cannot be the reason for this twist.

The mismatch of lattice parameters of 0.14 can lead to an experimentally obtained sign of the bending system. However, the experimentally determined values of the curvature radiuses (see Table I) are far from agreeing with the calculated values. To determine the reason for such disagreement we calculated the distribution of elastic deformations in the system as a function of thickness ${ }^{12}$ using

$$
\varepsilon(z)=-\varepsilon^{0}(z)+\int_{0}^{d} \varepsilon^{0}(z) d z+R^{-1}\left(z-\frac{d}{2}\right),
$$

where $z$ is the growth direction, $d$ is the thickness of the structure, $R$ is the curvature radius of the system, and $\varepsilon^{0}(z)$ is the internal deformation of the structure, given by

$$
\varepsilon^{0}(z)=\sum_{i=1}^{n-1} f^{(i)} \vartheta\left(z-z_{i}\right)
$$

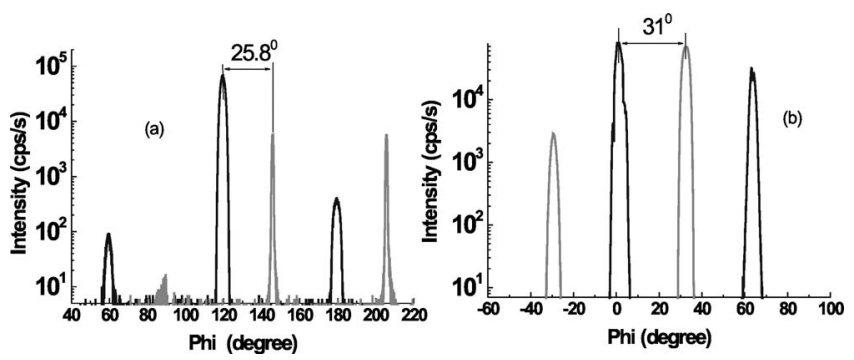

FIG. 2. $\phi$-scans around the direction normal to the surface of the diffraction

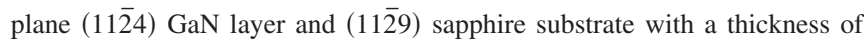
$450 \mu \mathrm{m}$ (a) and $3000 \mu \mathrm{m}$ (b). 

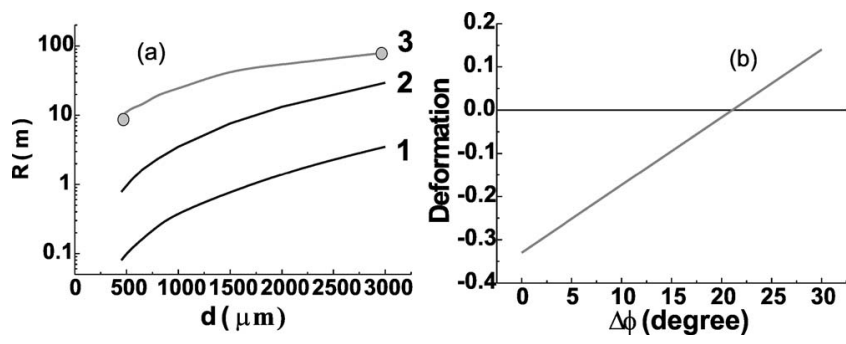

FIG. 3. (a) Dependence of curvature radius on substrate thickness: (1) ideal structure; (2) structure with uniform dislocation distribution on layer thickness; (3) structure accounting for twist between cells of GaN layer and sapphire substrate, circles-experimental results. (b) Deformation state variation as a function of twist angle between cells of GaN layer and sapphire substrate.

The calculation data allow the conclusion that in the case of a thick substrate the deformation jump at the interface is one order of magnitude smaller than in the case of a thin substrate. For GaN layers it remains practically the same in both cases. This obvious results in a much smaller dislocation density at the interface required to compensate the mismatch in the case of a thick substrate. This condition is confirmed experimentally (see Table I).

The experimental and theoretical dependencies of the curvature radius on substrate thickness are shown in Fig. 3(a). The curvature radiuses of the structures were calculated using the following expression:

$$
R^{-1}=\frac{12}{d^{3}} \int_{0}^{d} \varepsilon^{0}(z)\left(z-\frac{d}{2}\right) d z
$$

The additional bending contribution can be calculated ${ }^{13}$ using the relation of the dislocation density with the corresponding deformation, $\varepsilon^{0}(z)$ by the following equation:

$$
\alpha=\frac{R(d)-R_{m}}{R(d) R_{m}} \frac{d_{1}^{3}}{\left(3 d_{1}-2 d_{2}\right) d_{2}^{2}},
$$

where $R_{m}$ and $R(d)$ are the measured and the calculated curvature radius, respectively; $d_{1}$ and $d_{2}$ are the thickness of the layer and substrate, respectively; $\alpha$ is the dislocation density normalized by the Burgers vector. However, the dislocation densities required to compensate the strains caused by the difference between the system curvatures exceed $N_{D}=3.76$ $\times 10^{12} \mathrm{~cm}^{-2}$ for a thin substrate and $N_{D}=3.53 \times 10^{10} \mathrm{~cm}^{-2}$ for a thick substrate, which does not agree with the experimental data obtained from an analysis of the rocking curves. Previously it was demonstrated ${ }^{12,13}$ that the value of the deformation jump (curvature radius) depends on the character- istic distribution of dislocations in layers. Our calculations show that this fact does not allow us to find agreement with the changes in the curvature radius obtained experimentally. Thus, the large mismatch in bending structures cannot be explained only as a result of the dislocation contribution [curve 2, Fig. 3(a)]. To discover the reason for such a disagreement we calculated a contribution of volume fractions for every cell orientation to obtain a structure with variable curvature [Fig. 3(b)] (a planar structure of 0 curvature can be obtained at a ratio of $30 \%$ of the GaN volume with a mismatch $\Delta a / a=0.14$, and $70 \%$ of the volume with a mismatch $\Delta a / a=-0.33)$. Thus the system curvature can be regulated by twisting the GaN and sapphire cells. The twist by $25.8^{\circ}$ (less than is needed for a mismatch $\Delta a / a=0.14$ ) leads to a decrease of the deformation jump by $7 \%$.

In summary, the main difference between deformation parameters of heterostructures grown on thick and thin substrates is caused by the different values in the twists of hexagonal cells, with a relatively equivalent influence of the dislocation structure. These twists appear to be one of the channels of elastic strain relaxation in the system, because they lead to a reduction in bending curvature and corresponding macrodeformations.

${ }^{1}$ S. C. Jain, M. Willander, J. Narayan, and R. Van Overstraeten, J. Appl. Phys. 87, 965 (2000).

${ }^{2}$ C. Roder, S. Einfeldt, S. Figge, T. Paskova, D. Hommel, P. P. Paskov, B. Monemar, U. Behn, B. A. Haskell, P. T. Fini, and S. Nakamura, J. Appl. Phys. 100, 103511 (2006).

${ }^{3}$ B. Liu, R. Zhang, Z. L. Xie, H. Lu, Q. J. Liu, Z. Zhang, Y. Li, X. Q. Xiu, P. Chen, P. Han, S. L. Gu, Y. Shi, Y. D. Zheng, and W. J. Schaff, J. Appl. Phys. 103, 023504 (2008).

${ }^{4}$ L. Kirste, K. M. Pavlov, S. T. Mudie, V. I. Punegov, and N. Herres, J. Appl. Crystallogr. 38, 183 (2005).

${ }^{5}$ V. V. Ratnikov, R. N. Kyutt, T. V. Shubina, T. Paskova, and B. Monemar, J. Phys. D: Appl. Phys. 34, A30 (2001).

${ }^{6}$ V. P. Klad'ko, S. V. Chorneni'kii, A. V. Naumov, A. V. Komarov, M. Tacano, Yu. N. Sveshnikov, S. A. Vitusevich, and A. E. Belyaev, Semiconductors 40, 1060 (2006).

${ }^{7}$ M. A. Moram and M. E. Vickers, Rep. Prog. Phys. 72, 036502 (2009).

${ }^{8}$ P. Fewster, X-Ray Scattering from Semiconductors (Imperial College Press, London, 2000).

${ }^{9}$ G. K. Williamson and W. H. Hall, Acta Metall. 1, 22 (1953).

${ }^{10}$ V. P. Kladko, A. F. Kolomys, M. V. Slobodian, V. V. Strelchuk, V. G. Raycheva, A. E. Belyaev, S. S. Bukalov, H. Hardtdegen, V. A. Sydoruk, N. Klein, and S. A. Vitusevich, J. Appl. Phys. 105, 063515 (2009).

${ }^{11}$ V. Cimalla, J. Pezoldt, and O. Ambacher, J. Phys. D 40, 6386 (2007).

${ }^{12}$ Yu. A. Tkhorik and L. S. Khazan, Plastic Deformation and Misfit Dislocations in Heteroepitaxial Systems (in Russian) (Naukova Dumka, Kyiv, 1983), p. 304.

${ }^{13}$ V. P. Kladko, T. G. Kryshtab, G. N. Semenova, L. S. Khazan, and O. S Bashevskaya, Sov. Phys. Solid State 33, 1803 (1991). 